\title{
Two-parameter partially correlated ground-state electron density of some light spherical atoms from Hartree-Fock theory with nonintegral nuclear charge
}

\author{
Nicolás A. Cordero \\ Departamento de Física, Universidad de Burgos, C/ Villadiego s/n, E-09001 Burgos, Spain \\ Norman H. March \\ Department of Physics, University of Antwerp (RUCA). 171 Groenenborgerlaan, B-2020 Antwerp, Belgium \\ and Oxford University, Oxford, England \\ Julio A. Alonso \\ Departamento de Física Teórica, Atómica y Óptica, Universidad de Valladolid, E-47011 Valladolid, Spain
}

(Received 31 January 2007; published 7 May 2007)

\begin{abstract}
Partially correlated ground-state electron densities for some spherical light atoms are calculated, into which nonrelativistic ionization potentials represent essential input data. The nuclear cusp condition of Kato is satisfied precisely. The basic theoretical starting point, however, is Hartree-Fock (HF) theory for the $N$ electrons under consideration but with nonintegral nuclear charge $Z^{\prime}$ slightly different from the atomic number $Z$ $(=N)$. This HF density is scaled with a parameter $\lambda$, near to unity, to preserve normalization. Finally, some tests are performed on the densities for the atoms $\mathrm{Ne}$ and $\mathrm{Ar}$, as well as for $\mathrm{Be}$ and $\mathrm{Mg}$.
\end{abstract}

DOI: 10.1103/PhysRevA.75.052502

PACS number(s): 31.10. $+\mathrm{z}, 31.15 . \mathrm{Ar}, 31.25 . \mathrm{Eb}$

\section{INTRODUCTION}

In their celebrated theoretical study about adding Coulomb correlation effects to the Hartree-Fock (HF) approximation, Møller and Plesset [1] concluded by emphasizing the accuracy of the HF ground-state electron density $\rho(\mathbf{r})$ in atomic physics. Their specific conclusion was that $\rho(\mathbf{r})$ was correct to second order in the difference between the correct nonrelativistic Hamiltonian and the Fock operator. Motivated by this Møller-Plesset result we reopen here the question of going beyond the HF ground-state density for some closed shell atoms. We start out from an unconventional use of the HF method with nonintegral nuclear charge — say, $Z^{\prime}$ — in order to get the nonrelativistic ionization potential and scale the resulting density to satisfy Kato's cusp condition for the electron density at the nucleus [2].

The numerically calculated $\mathrm{HF}$ ground-state density for species with nuclear charge $Z^{\prime}$ and $N$ electrons, $\rho_{\mathrm{HF}}\left(Z^{\prime}, N, r\right)$, is well known to have an exponential factor in its decay at large distances from the nucleus, taken as origin $r=0$, of the form

$$
\rho_{\mathrm{HF}}\left(Z^{\prime}, N, r\right) \sim \exp \left[-2 \sqrt{2 I_{\mathrm{K}}\left(Z^{\prime}, N\right)} r\right],
$$

in atomic units, where $I_{\mathrm{K}}\left(Z^{\prime}, N\right)$ is the appropriate Koopmans' ionization potential, which in turn is the one-electron $\mathrm{HF}$ eigenvalue associated with the highest occupied atomic orbital (HOAO). In contrast, for a neutral atom with $N=Z$, the exact exponential decay factor of the nonrelativistic ground-state electron density $\rho_{\mathrm{NR}}(r)$ is known to be [3]

$$
\rho_{\mathrm{NR}}(Z, N, r) \sim \exp \left[-2 \sqrt{2 I_{\mathrm{NR}}(Z, N)} r\right],
$$

where $I_{\mathrm{NR}}(Z, N)$ is the nonrelativistic ionization potential of the neutral atom with atomic number $Z$ under consideration. Thus in Sec. II below we summarize an available method which allows $I_{\mathrm{NR}}$ to be determined semiempirically. With this nonrelativistic input information, we describe the basic scaling proposal of this work. This will enable us to connect uniquely the basic $\mathrm{HF}$ theoretical calculation of $\rho_{\mathrm{HF}}\left(Z^{\prime}, N, r\right)$ cited in Eq. (1) to the desired (partially correlated) groundstate density $\rho_{\mathrm{NR}}(Z=N, r)$ of the neutral atom, cited in Eq. (2). This section ends by analyzing our results of the twoparameter $\rho_{\mathrm{NR}}(Z=N, r)$ proposed for Ne and Ar. Section III compares and contrasts these results for rare gas atoms with the divalent atoms $\mathrm{Be}$ and $\mathrm{Mg}$ chosen because they also have spherical electron densities. Section IV gives some tests of the proposed densities by comparison with quantum simulation studies. A summary together with proposals for future studies which should prove fruitful constitutes Sec. V.

\section{SCALING APPROACH TO FORM CORRELATED GROUND-STATE ELECTRON DENSITIES FROM HARTREE-FOCK DENSITIES FOR Ne AND Ar}

For a nonrelativistic atom with atomic number $Z$ and $N$ $(=Z)$ electrons, the electronic density far from the nucleus decays as in Eq. (2). Within the Hartree-Fock approximation, the electronic density of a fictitious atom with atomic number $Z^{\prime}$ and $N$ electrons falls off exponentially as given by Eq. (1), where $I_{\mathrm{K}}\left(Z^{\prime}, N\right)$ is its ionization potential calculated using Koopmans' theorem [i.e., the eigenvalue of the highest occupied atomic orbital, with the opposite sign $\left.I_{\mathrm{K}}\left(Z^{\prime}, N\right)=-\varepsilon_{\mathrm{HOAO}}\left(Z^{\prime}, N\right)\right]$.

If we scale this HF density using the norm-conserving ansatz

$$
\rho_{\lambda}\left(Z^{\prime}, N, r\right)=\lambda^{3} \rho_{\mathrm{HF}}\left(Z^{\prime}, N, \lambda r\right),
$$

where $\lambda$ is a positive parameter, the asymptotic behavior of the scaled density will be

$$
\rho_{\lambda}\left(Z^{\prime}, N, r\right) \sim \exp \left[-2 \sqrt{2 I_{\mathrm{K}}\left(Z^{\prime}, N\right)} \lambda r\right] .
$$

If we require the scaled density to decay in its long-range behavior like the exact nonrelativistic one, $\rho_{\lambda}\left(Z^{\prime}, N, r\right)$ 
TABLE I. Ionization potentials (in a.u.) and fitted parameters for the atoms studied. The experimental ionization potentials $I_{\text {expt }}(Z, N)$ are taken from [5] and the nonrelativistic ones $I_{\mathrm{NR}}(Z, N)$ are calculated according to Eq. (10). $I_{\mathrm{K}}(Z, N)$ and $I_{\mathrm{K}}\left(Z^{\prime}, N\right)$ are Koopmans' ionization potential for the atom with its original and slightly modified nuclear charge, respectively. In all cases $N=Z$.

\begin{tabular}{lccccccc}
\hline \hline Atom & $Z$ & $I_{\mathrm{K}}(Z, N)$ & $I_{\text {expt }}(Z, N)$ & $I_{\mathrm{NR}}(Z, N)$ & $Z^{\prime}$ & $\lambda=Z / Z^{\prime}$ & $I_{\mathrm{K}}\left(Z^{\prime}, N\right)$ \\
\hline $\mathrm{Ne}$ & 10 & 0.850410 & 0.792482 & 0.794464 & 9.9128 & 1.0088 & 0.780612 \\
$\mathrm{Ar}$ & 18 & 0.591017 & 0.579155 & 0.582173 & 17.9802 & 1.0011 & 0.580883 \\
\hline $\mathrm{Be}$ & 4 & 0.309270 & 0.342603 & 0.344332 & 4.1270 & 0.96922 & 0.366550 \\
$\mathrm{Mg}$ & 12 & 0.253053 & 0.280994 & 0.280740 & 12.0960 & 0.99207 & 0.285245 \\
\hline \hline
\end{tabular}

$\sim \rho_{\mathrm{NR}}(Z, N, r)$, we must impose the condition

$$
\sqrt{I_{\mathrm{K}}\left(Z^{\prime}, N\right)} \lambda=\sqrt{I_{\mathrm{NR}}(Z, N)} .
$$

On the other hand, if we want this scaled density to satisfy Kato's cusp condition at the nucleus [2] for the correct nuclear charge $Z$, we must have

$$
\begin{aligned}
\left.\frac{\partial \rho_{\lambda}\left(Z^{\prime}, N, r\right)}{\partial r}\right|_{r=0} & =-\frac{2 Z}{a_{0}} \rho_{\lambda}\left(Z^{\prime}, N, r=0\right) \\
& =-\frac{2 Z}{a_{0}} \lambda^{3} \rho_{\mathrm{HF}}\left(Z^{\prime}, N, r=0\right),
\end{aligned}
$$

and taking into account that

$$
\begin{aligned}
\left.\frac{\partial \rho_{\lambda}\left(Z^{\prime}, N, r\right)}{\partial r}\right|_{r=0} & =\left.\lambda^{3} \frac{\partial \rho_{\mathrm{HF}}\left(Z^{\prime}, N, \lambda r\right)}{\partial r}\right|_{r=0} \\
& =\left.\lambda^{4} \frac{\partial \rho_{\mathrm{HF}}\left(Z^{\prime}, N, r\right)}{\partial r}\right|_{r=0} \\
& =-\lambda^{4} \frac{2 Z^{\prime}}{a_{0}} \rho_{\mathrm{HF}}\left(Z^{\prime}, N, r=0\right),
\end{aligned}
$$

we obtain

$$
Z^{\prime} \lambda=Z \text {. }
$$

This identity shows that the two parameters of the model are not independent. This means that our two-parameter model is in fact a one-parameter model. Eliminating $\lambda$ between Eqs. (5) and (8) one finds

$$
\frac{I_{\mathrm{K}}\left(Z^{\prime}, N\right)}{Z^{\prime 2}}=\frac{I_{\mathrm{NR}}(Z, N)}{Z^{2}} .
$$

If we choose $Z^{\prime}$ so that identity (9) is satisfied, we have a density $\rho_{\lambda}\left(Z^{\prime}, N, r\right)$ that has the correct nonrelativistic behavior both at the nucleus and very far from it, and so it must include some amount of Coulomb correlation and can be considered as an approximation to $\rho_{\mathrm{NR}}(Z, N, r)$.

In order to calculate the nonrelativistic ionization potentials for $\mathrm{Ne}$ and $\mathrm{Ar}$ we have taken the experimental first ionization energies and made use of the relativistic corrections given by Chakravorty et al. [4]:

$$
I_{\mathrm{NR}}(Z, N)=I_{\text {expt }}(Z, N) \frac{R_{\infty}}{R_{Z}}+\Delta E_{\text {rel }}(Z, N),
$$

where

$$
\frac{R_{\infty}}{R_{Z}}=1+\frac{m_{e}}{M_{Z}}=1+\frac{m_{e}}{A-Z m_{e}}
$$

with $M_{Z}$ the nuclear mass, $m_{e}$ the electron mass, and $A$ the atomic mass. All the experimental values were taken from [5], and the nonrelativistic ionization potentials calculated according to Eq. (10) appear in Table I.

We have slightly modified the Froese-Fischer HF code $[6,7]$ to allow for noninteger atomic numbers and compute total densities in order to calculate $\rho_{\mathrm{HF}}(Z, N, r)$ and $\rho_{\lambda}\left(Z^{\prime}, N, r\right)$. Table I also includes the fitted parameters $Z^{\prime}$ and $\lambda$ for the spherical atoms $\mathrm{Ne}$ and Ar, as well as the Koopmans' ionization potential for the atom with its original $I_{\mathrm{K}}(Z, N)$ and slightly modified $I_{\mathrm{K}}\left(Z^{\prime}, N\right)$ nuclear charge. The latter is closer to $I_{\mathrm{NR}}(Z, N)$ but not identical to it since $Z^{\prime}$ $\neq Z$ and Eq. (9) must be satisfied. It is interesting to note that $Z^{\prime}$ is slightly less than $Z$ for these rare gas atoms, the difference between then being smaller for the heavier atom. This will be contrasted in Sec. III with the situation in Be and $\mathrm{Mg}$.

Figure 1 shows the scaled Hartree-Fock radial densities $4 \pi r^{2} \rho_{\lambda}\left(Z^{\prime}, N, r\right)$ for $\mathrm{Ne}$ and Ar. Both radial densities $4 \pi r^{2} \rho_{\lambda}\left(Z^{\prime}, N, r\right)$ and $4 \pi r^{2} \rho_{\mathrm{HF}}(Z, N, r)$ are very similar. In order to appreciate the small difference between them, Figs. 2 and 3 show $\Delta\left(4 \pi r^{2} \rho\right)=4 \pi r^{2} \rho_{\lambda}\left(Z^{\prime}, N, r\right)-4 \pi r^{2} \rho_{\mathrm{HF}}(Z, N, r)$ for $\mathrm{Ne}$ and $\mathrm{Ar}$, respectively. In both cases $\rho_{\lambda}\left(Z^{\prime}, N, r\right)$ $<\rho_{\mathrm{HF}}(Z, N, r)$ close to the nucleus and, of course, $\int_{0}^{\infty} \Delta\left(4 \pi r^{2} \rho\right) d r=0$ since the rescaling given by Eq. (3) is norm conserving, but there are two differences: $\Delta\left(4 \pi r^{2} \rho\right)$ has one oscillation for $\mathrm{Ne}$ but two for Ar and is one order of magnitude smaller for the latter.

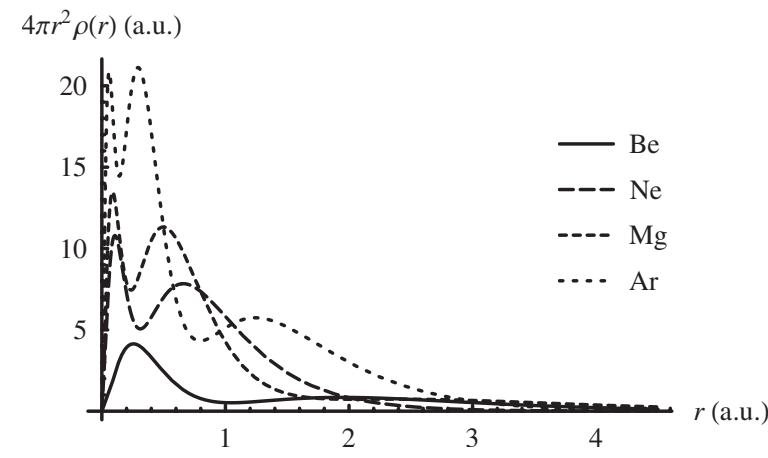

FIG. 1. Scaled Hartree-Fock radial densities in atomic units. 


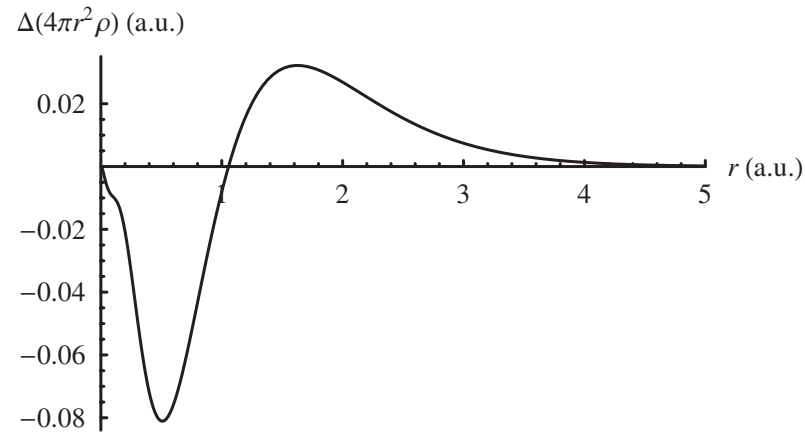

FIG. 2. Radial density difference (in atomic units) between the scaled and original HF densities for the $\mathrm{Ne}$ atom.

\section{COMPARISON BETWEEN RARE GAS ATOMS AND THE DIVALENT ELEMENTS Be AND Mg}

The purpose of this section is to present partially correlated ground-state electron densities for $\mathrm{Be}$ and $\mathrm{Mg}$ and to compare and contrast with the results for $\mathrm{Ne}$ and Ar. For the latter purpose, we have added to Table I the values of $\lambda$ and $Z^{\prime}$ for $\mathrm{Be}$ and $\mathrm{Mg}$ and the corresponding scaled radial densities to Fig. 1. The most important difference to be emphasized is that whereas, as stressed in Sec. II, the starting HF densities for $\mathrm{Ne}$ and Ar apply to "weakly negative" atomic ions, the opposite situation obtains for the "divalent" elements $\mathrm{Be}$ and $\mathrm{Mg}$. While the values of the normalization scaling parameter $\lambda$ are all within a few percent of unity, for $\mathrm{Ne}$ and $\mathrm{Ar}, \lambda$ is slightly greater than 1 , while for $\mathrm{Be}$ and $\mathrm{Mg}$, $\lambda$ is slightly smaller than 1 . However, with regard to the $\lambda$ variation there is a common feature for the four atoms: $Z^{\prime}$ tends to $Z$ in such a way that $|\lambda-1|$ decreases monotonically with $Z$. In order to understand this result we must take into account that, although the Coulomb correlation contribution to the total energy of light neutral atoms increases roughly linearly with the number of pairings between antiparallel spin electrons (and thus with the atomic number) $[8,9]$, the correlation contribution to the ionization potential is not a monotonic increasing function of $Z[10]$. For the four atoms considered here the last contribution is similar [10] and $\lambda$ must get closer to unity as $Z$ increases; otherwise, the correlation contribution to the ionization potential should increase with $Z$, since the number of electrons increases.

All this means that the correlation effects become less important in correcting HF properties for the atoms consid-

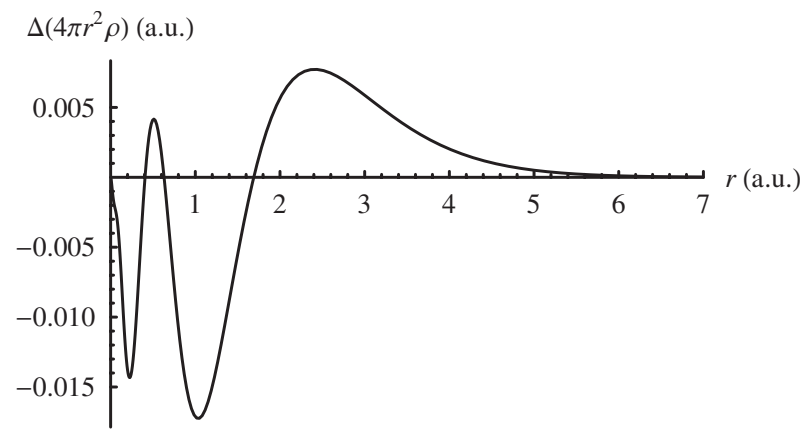

FIG. 3. Radial density difference (in atomic units) between the scaled and original HF densities for the Ar atom.

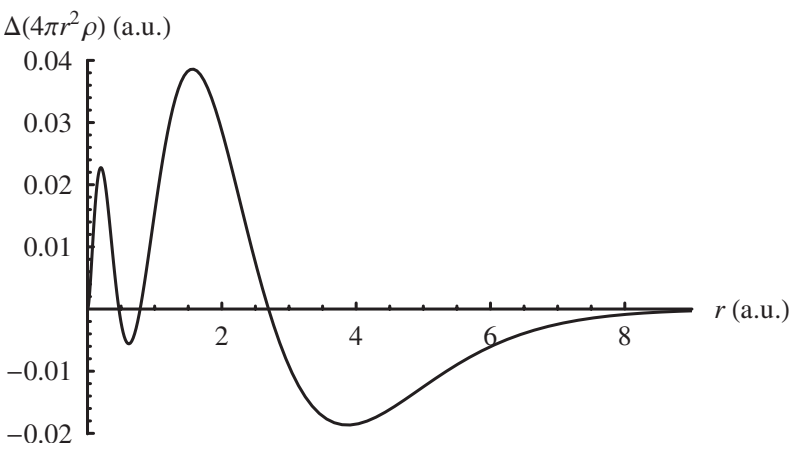

FIG. 4. Radial density difference (in atomic units) between the scaled and original HF densities for the Be atom.

ered as the atomic number $Z$ increases. Thus, Be exhibits the largest deviations from $\mathrm{HF}$ results, as shown clearly in the departures of $Z^{\prime}$ and $\lambda$ from $Z$ and 1, respectively. Again, as with $\mathrm{Ne}$ and Ar displayed in Sec. II, radial density differences are also shown for Be and $\mathrm{Mg}$ in Figs. 4 and 5. In this case $\rho_{\lambda}\left(Z^{\prime}, N, r\right)>\rho_{\mathrm{HF}}(Z, N, r)$, close to the nucleus, in contrast to what happened for $\mathrm{Ne}$ and $\mathrm{Ar}$ (owing to the different sign of $\lambda-1)$ and $\Delta\left(4 \pi r^{2} \rho\right)$ has two oscillations for both Be and $\mathrm{Mg}$.

\section{TESTS OF THE PROPOSED DENSITIES}

Tables II-V present the density at the nucleus and the moments of the density calculated using the original HartreeFock density $\rho_{\mathrm{HF}}(Z, N, r)$ as well as the scaled one $\rho_{\lambda}\left(Z^{\prime}, N, r\right)$ for the four atoms under consideration. Monte Carlo and accurate configuration interaction (CI) results from different authors [11-14] are given for comparison. The mean relative error

$$
\overline{\Delta_{\text {rel }}\left\langle r^{n}\right\rangle}=\frac{1}{n_{\max }+2} \sum_{\substack{n=-2 \\ n \neq 0}}^{n_{\max }} \frac{\left\langle r^{n}\right\rangle-\left\langle r^{n}\right\rangle_{\text {accurate }}}{\left\langle r^{n}\right\rangle_{\text {accurate }}}
$$

and the mean relative error in absolute value,

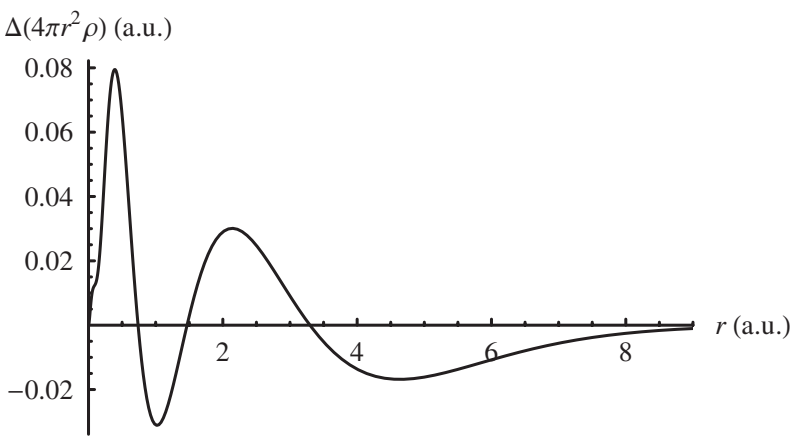

FIG. 5. Radial density difference (in atomic units) between the scaled and original $\mathrm{HF}$ densities for the $\mathrm{Mg}$ atom. 
TABLE II. Density at the nucleus and moments of the density (in a.u.) for the Be atom.

\begin{tabular}{lccccccc}
\hline \hline & $\mathrm{HF}$ & $\mathrm{Scaled} \mathrm{HF}$ & $\mathrm{VMC}^{\mathrm{a}}$ & $\mathrm{DMC}^{\mathrm{b}}$ & $\left.\mathrm{VMC}^{\mathrm{v}}\right)^{\mathrm{c}}$ & $\left.\mathrm{VMC}^{\mathrm{Ne}}\right)^{\mathrm{d}}$ & $\mathrm{ECG}^{\mathrm{e}}$ \\
\hline$\rho(0)$ & 35.388 & 35.654 & $34.665(13)$ & $35.32(8)$ & $35.8(3)$ & $35.3(1)$ & 35.3116 \\
\hline$\left\langle r^{-2}\right\rangle$ & 57.618 & 58.001 & $56.79(2)$ & $57.40(10)$ & $58.3(5)$ & $57.4(2)$ & 57.59808 \\
$\left\langle r^{-1}\right\rangle$ & 8.4088 & 8.4595 & $8.3734(14)$ & $8.403(4)$ & $8.408(2)$ & $8.433(2)$ & 8.42735 \\
$\langle r\rangle$ & 6.1288 & 5.9766 & $5.9841(5)$ & $6.065(7)$ & $6.217(2)$ & $5.985(1)$ & 5.97256 \\
$\left\langle r^{2}\right\rangle$ & 17.319 & 16.330 & $16.197(3)$ & $16.79(4)$ & $18.154(8)$ & $16.343(9)$ & 16.2476 \\
$\left\langle r^{3}\right\rangle$ & 63.151 & 57.454 & & & $69.57(4)$ & $57.43(6)$ & 56.772 \\
$\left\langle r^{4}\right\rangle$ & 270.66 & 237.12 & & & $318.2(5)$ & $236.7(4)$ & 232.12 \\
\hline$\overline{\Delta_{\text {rel }}\left\langle r^{n}\right\rangle}$ & $6.14 \%$ & $0.84 \%$ & $-0.54 \%$ & $1.06 \%$ & $12.74 \%$ & $0.61 \%$ & \\
\hline$\Delta_{\text {rel }}\left\langle r^{n}\right\rangle \mid$ & $6.22 \%$ & $0.84 \%$ & $0.64 \%$ & $1.38 \%$ & $12.82 \%$ & $0.72 \%$ & \\
\hline \hline
\end{tabular}

${ }^{\mathrm{a}}$ Variational Monte Carlo results from Langfelder et al. [11].

${ }^{\mathrm{b}}$ Diffusion Monte Carlo results from Langfelder et al. [11].

${ }^{c}$ Variance-optimized variational Monte Carlo results from Gálvez et al. [12].

${ }^{\mathrm{d}}$ Energy-optimized variational Monte Carlo results from Gálvez et al. [12].

${ }^{\mathrm{e}}$ Accurate results from Komasa et al. [13] using exponentially correlated Gaussian functions.

$$
\overline{\left|\Delta_{\text {rel }}\left\langle r^{n}\right\rangle\right|}=\frac{1}{n_{\max }+2} \sum_{\substack{n=-2 \\ n \neq 0}}^{n_{\max }}\left|\frac{\left\langle r^{n}\right\rangle-\left\langle r^{n}\right\rangle_{\text {accurate }}}{\left\langle r^{n}\right\rangle_{\text {accurate }}}\right|,
$$

appear at the bottom of the tables.

HF results consistently overestimate the moments of the density for $\mathrm{Be}, \mathrm{Mg}$, and $\operatorname{Ar}\left(\overline{\Delta_{\text {rel }}\left\langle r^{n}\right\rangle} \approx\left|\Delta_{\text {rel }}\left\langle r^{n}\right\rangle\right|\right)$ while they underestimate them for $\mathrm{Ne}\left(\overline{\Delta_{\text {rel }}\left\langle r^{n}\right\rangle} \approx-\left|\overline{\Delta_{\text {rel }}}\left\langle r^{n}\right\rangle\right|\right)$. In the case of $\mathrm{Be}$ (for which the greatest number of accurate moments are available in the literature) our scaled density consistently overestimates the moments but improves the results by roughly one order of magnitude. This improvement is not homogeneous; there is a slight worsening on $\left\langle r^{-2}\right\rangle$ and $\left\langle r^{-1}\right\rangle$ and a big improvement for higher-order moments. For the rest of the atoms considered the scaled densities do not ex- hibit an overall underestimation or overestimation of the moments $\left(\left|\overline{\Delta_{\text {rel }}\left\langle r^{n}\right\rangle \mid}\right| \neq\left|\Delta_{\text {rel }}\left\langle r^{n}\right\rangle\right|\right)$. There is, however, a common trend: although scaled HF values are better than HF ones (except for Ar), they modestly improve (or slightly worsen) low-order moments while clearly improve high-order ones. This seems to imply that scaled densities are better than HF ones overall but perform better far from the nucleus than close to it. Regarding the comparison with Monte Carlo results, scaled HF results are clearly competitive with them.

Liu et al. $[15,16]$ have shown that the density at the nucleus of an atom is in fact a nonlocal functional of the electron density that can be calculated via four different integrals:

$$
\rho(0)_{1}=-\frac{1}{4 \pi} \int \frac{\nabla^{2} \rho(\mathbf{r})}{|\mathbf{r}|} d \mathbf{r}
$$

TABLE III. Density at the nucleus and moments of the density (in a.u.) for the Ne atom.

\begin{tabular}{lccccccr}
\hline \hline & HF & Scaled HF & VMC $^{\mathrm{a}}$ & $\mathrm{DMC}^{\mathrm{b}}$ & $\left.\mathrm{VMC}^{\mathrm{v}}\right)^{\mathrm{c}}$ & $\left.\mathrm{VMC}^{\mathrm{e}}\right)^{\mathrm{d}}$ & MR-SDCI $^{\mathrm{e}}$ \\
\hline$\rho(0)$ & 619.92 & 619.37 & $609.83(17)$ & $619.0(9)$ & $615(2)$ & $621(2)$ & \\
\hline$\left\langle r^{-2}\right\rangle$ & 414.89 & 414.35 & $409.69(12)$ & $414.1(4)$ & $412 .(2)$ & $417 .(2)$ & 414.8753 \\
$\left\langle r^{-1}\right\rangle$ & 31.113 & 31.032 & $30.883(4)$ & $31.03(2)$ & $31.128(3)$ & $31.134(5)$ & 31.1093 \\
$\langle r\rangle$ & 7.8911 & 7.9590 & $7.9844(5)$ & $7.974(6)$ & $7.935(1)$ & $7.903(1)$ & 7.9385 \\
$\left\langle r^{2}\right\rangle$ & 9.3718 & 9.5677 & $9.6640(13)$ & $9.64(2)$ & $9.567(3)$ & $9.468(2)$ & 9.5565 \\
$\left\langle r^{3}\right\rangle$ & 14.383 & 14.910 & & & $15.13(1)$ & $14.94(2)$ & \\
$\left\langle r^{4}\right\rangle$ & 27.188 & 28.709 & & & & & \\
\hline$\overline{\Delta_{\text {rel }}\left\langle r^{n}\right\rangle}$ & $-0.63 \%$ & $0.00 \%$ & $-0.07 \%$ & $0.22 \%$ & $-0.14 \%$ & $-0.20 \%$ & \\
\hline$\Delta_{\text {rel }}\left\langle r^{n}\right\rangle \mid$ & $0.64 \%$ & $0.19 \%$ & $0.92 \%$ & $0.44 \%$ & $0.23 \%$ & $0.49 \%$ & \\
\hline \hline
\end{tabular}

${ }^{\mathrm{a}}$ Variational Monte Carlo results from Langfelder et al. [11].

${ }^{\mathrm{b}}$ Diffusion Monte Carlo results from Langfelder et al. [11].

${ }^{\mathrm{c}}$ Variance-optimized variational Monte Carlo results from Gálvez et al. [12].

${ }^{\mathrm{d}}$ Energy-optimized variational Monte Carlo results from Gálvez et al. [12].

${ }^{\mathrm{e}}$ Results from Meyer et al. [14] using multireference singly and doubly excited configuration interaction that recovers $95.7 \%$ of the total correlation energy. 
TABLE IV. Density at the nucleus and moments of the density (in a.u.) for the $\mathrm{Mg}$ atom.

\begin{tabular}{lccr}
\hline \hline & HF & Scaled HF & MR-SDCI $^{\mathrm{a}}$ \\
\hline$\rho(0)$ & 1093.7 & 1094.8 & \\
\hline$\left\langle r^{-2}\right\rangle$ & 614.85 & 615.63 & 614.7719 \\
$\left\langle r^{-1}\right\rangle$ & 39.920 & 40.012 & 39.9303 \\
$\langle r\rangle$ & 12.258 & 12.058 & 12.1357 \\
$\left\langle r^{2}\right\rangle$ & 29.612 & 28.189 & 28.5698 \\
$\left\langle r^{3}\right\rangle$ & 114.30 & 104.83 & \\
$\left\langle r^{4}\right\rangle$ & 555.24 & 490.44 & \\
\hline$\overline{\Delta_{\text {rel }}\left\langle r^{n}\right\rangle}$ & $1.16 \%$ & $-0.41 \%$ & \\
\hline$\Delta_{\text {rel }}\left\langle r^{n}\right\rangle \mid$ & $1.17 \%$ & $0.58 \%$ & \\
\hline \hline
\end{tabular}

${ }^{a}$ Results from Meyer et al. [14] using multireference singly and doubly excited configuration interaction that recovers $93.7 \%$ of the total correlation energy.

$$
\begin{gathered}
\rho(0)_{2}=-\frac{1}{4 \pi} \int \frac{\mathbf{r} \cdot \nabla \rho(\mathbf{r})}{|\mathbf{r}|^{3}} d \mathbf{r}, \\
\rho(0)_{3}=-\frac{1}{4 \pi} \int \frac{e^{-\alpha|\mathbf{r}|}}{|\mathbf{r}|}\left[\nabla^{2} \rho(\mathbf{r})-\alpha^{2} \rho(\mathbf{r})\right] d \mathbf{r}, \\
\rho(0)_{4}=-\frac{1}{4 \pi} \int \frac{e^{-\beta|\mathbf{r}|}}{|\mathbf{r}|^{2}}\left[\frac{\mathbf{r} \cdot \nabla \rho(\mathbf{r})}{|\mathbf{r}|}-\beta \rho(\mathbf{r})\right] d \mathbf{r},
\end{gathered}
$$

where $\alpha$ and $\beta$ are arbitrary positive constants. If we particularize for a spherical atom $\rho(\mathbf{r})=\rho(r)$, these four identities read

$$
\begin{gathered}
\rho(0)_{1}=-\int_{0}^{\infty}\left[r \rho^{\prime \prime}(r)+2 \rho^{\prime}(r)\right] d r, \\
\rho(0)_{2}=-\int_{0}^{\infty} \rho^{\prime}(r) d r, \\
\rho(0)_{3}=-\int_{0}^{\infty} e^{-\alpha r}\left[r \rho^{\prime \prime}(r)+2 \rho^{\prime}(r)-\alpha^{2} r \rho(r)\right] d r, \\
\rho(0)_{4}=-\int_{0}^{\infty} e^{-\beta r}\left[\rho^{\prime}(r)-\beta \rho(r)\right] d r,
\end{gathered}
$$

where $\rho^{\prime}(r)$ and $\rho^{\prime \prime}(r)$ indicate the first and second derivatives of the density with respect to $r$.

Liu et al. [15] tested these identities using the HF densities of Clementi and Roetti [17] obtaining an excellent agreement between values for these expressions and densities at the nucleus. Our results show that this agreement is maintained (within our numerical accuracy) for scaled HF densities. This is not surprising since, as has been noted by Liu et al. [16], Eqs. (16) and (17) demonstrate that the density at the origin $\rho(0)$ is homogeneous of degree 3 with respect to coordinate scaling, just like the electron density $\rho(r)$ itself.
TABLE V. Density at the nucleus and moments of the density (in a.u.) for the Ar atom.

\begin{tabular}{lccr}
\hline \hline & HF & Scaled HF & MR-SDCI $^{\mathrm{a}}$ \\
\hline$\rho(0)$ & 3839.8 & 3839.4 & \\
\hline$\left\langle r^{-2}\right\rangle$ & 1465.0 & 1464.8 & 1464.9102 \\
$\left\langle r^{-1}\right\rangle$ & 69.725 & 69.704 & 69.7426 \\
$\langle r\rangle$ & 16.071 & 16.097 & 16.0382 \\
$\left\langle r^{2}\right\rangle$ & 26.034 & 26.144 & 25.9344 \\
$\left\langle r^{3}\right\rangle$ & 55.988 & 56.397 & \\
$\left\langle r^{4}\right\rangle$ & 144.79 & 146.36 & \\
\hline$\overline{\Delta_{\text {rel }}\left\langle r^{n}\right\rangle}$ & $0.14 \%$ & $0.28 \%$ & \\
\hline$\Delta_{\text {rel }}\left\langle r^{n}\right\rangle \mid$ & $0.15 \%$ & $0.31 \%$ & \\
\hline \hline
\end{tabular}

${ }^{\mathrm{a}}$ Results from Meyer et al. [14] using multireference singly and doubly excited configuration interaction that recovers $91.4 \%$ of the total correlation energy.

This fact does not mean that our scaled densities at the origin for the atoms studied are $\lambda^{3}$ times the HF ones (as can be checked using the data in Tables II-V) because we have modified the nuclear charge in order to recover the nonrelativistic ionization potential of the neutral atom.

\section{SUMMARY AND FUTURE DIRECTIONS}

The main content of this work is the use of the HF ground-state density $\rho_{\mathrm{HF}}\left(Z^{\prime}, N, r\right)$ as the starting point from which to construct partially correlated densities $\rho(Z=N, r)$ for especially the rare gas atoms $\mathrm{Ne}$ and Ar. This is achieved via two parameters. The first one is the nonintegral nuclear charge $Z^{\prime}$ for chosen $N$ (10 for $\mathrm{Ne}$ and 18 for Ar). Table I shows that $Z^{\prime}$ is slightly less than 10 and 18 for $\mathrm{Ne}$ and Ar, respectively, The second one is a scaling parameter $\lambda$ (very near to unity in accord with the expectations of Møller and Plesset [1]). Choosing these parameters in order to reproduce (a) the nonrelativistic ionization potential and (b) Kato's nuclear cusp condition [2] leads to a relationship between them turning in fact our two-parameter model into a single parameter one.

Comparison with the quantal simulation data [e.g., diffusion quantum Monte Carlo (QMC)] supports the quality of the partially correlated densities for $\mathrm{Ne}$ and $\mathrm{Ar}$ presented here. However, the proposed density still needs some modest refinement close to the nucleus and three methods will be mentioned below which may prove useful for future studies. Before that, we have thought it of interest to compare the atoms $\mathrm{Be}$ and $\mathrm{Mg}$, which as for $\mathrm{Ne}$ and $\mathrm{Ar}$ also have spherical electron densities, with the rare gas atoms. One interesting difference is that the HF starting point, as seen in Table I, is now from (slightly) positive ions (i.e., $Z>4$ for $\mathrm{Be}$ and $Z>12$ for $\mathrm{Mg}$ ) in contrast to the weak nonintegral negative ions for $\mathrm{Ne}$ and Ar.

As to future directions, it seems important to refine the method presented here for obtaining partially correlated densities to improve the nonrelativistic electron density $\rho(0)$ at the (assumed) point nucleus. At least three approaches then 
offer promise. The first is associated with the Hiller-SucherFeinberg (HSF) identity (see, for example, Cioslowski et al. $[18,19]$ and Holas and March [20], the latter work casting the HSF study in the general context of density functional theory). Particularly the work of Cioslowski et al. points to the possible use of the HSF identity in refining $\rho(0)$. The other is the older study of "bounds" of $\rho(0)$ by HoffmannOstenhof et al. [3,21]. The third and most recent study is that of Liu [16] who has shown that the electron density of a spherical atom must satisfy some integral rules that involve the atomic number $Z$. Besides, analysis of the phase equation of Hartree-Fock theory [22,23] could give some insight into the differences between HF and scaled HF densities. Also, for the future, generalizations of the present approach to "rather spherical" molecules like $\mathrm{SiH}_{4}$ and $\mathrm{GeH}_{4}$ (see, e.g., the early work of March [24]) would seem worthwhile, es- pecially based on $\mathrm{HF}$ calculations on $\mathrm{SiH}_{4}$ such as those of Carter [25].

\section{ACKNOWLEDGMENTS}

We gratefully acknowledge financial support from Ministerio de Educación y Ciencia (project MAT2005-06544-C03) and Junta de Castilla y León (grant VA039A05). N.H.M. wishes to acknowledge that his contribution to this study was brought to fruition during a stay at the University of Valladolid. He thanks Professors J. A. Alonso and M. Santander for generous hospitality. Finally N.H.M. wishes to acknowledge a most valuable discussion and subsequent correspondence with Professor J. D. Talman on the general area embraced by this present article.
[1] C. Møller and M. S. Plesset, Phys. Rev. 46, 618 (1934).

[2] T. Kato, Commun. Pure Appl. Math. 10, 151 (1957).

[3] T. Hoffmann-Ostenhof, M. Hoffmann-Ostenhof, and R. Ahlrichs, Phys. Rev. A 18, 328 (1978).

[4] S. J. Chakravorty, S. R. Gwaltney, E. R. Davidson, F. A. Parpia, and C. F. Fischer, Phys. Rev. A 47, 3649 (1993).

[5] CRC Handbook of Chemistry and Physics, edited by D. R. Lide (CRC Press, Boca Raton, 2003).

[6] C. Froese-Fischer, Comput. Phys. Commun. 43, 355 (1987).

[7] G. Gaigalas and C. Froese-Fischer, Comput. Phys. Commun. 98, 255 (1996).

[8] J. A. Alonso and N. A. Cordero, J. Phys. B 29, 1629 (1996).

[9] J. A. Alonso, N. H. March, N. A. Cordero, and A. Rubio, J. Phys. B 36, 2695 (2003).

[10] N. A. Cordero, O. V. Gritsenko, A. Rubio, L. C. Balbás, and J. A. Alonso, Int. J. Quantum Chem. 52, 993 (1994).

[11] P. Langfelder, S. M. Rothstein, and J. Vrbikb, J. Chem. Phys. 107, 8525 (1997).

[12] F. J. Gálvez, E. Buendía, and A. Sarsa, J. Chem. Phys. 115, 1166 (2001).
[13] J. Komasa, W. Cencek, and J. Rychlewski, Phys. Rev. A 52, 4500 (1995).

[14] H. Meyer, T. Miiller, and A. Schweig, J. Mol. Struct.: THEOCHEM 360, 55 (1996).

[15] S. Liu, R. G. Parr, and A. Nagy, Phys. Rev. A 52, 2645 (1995).

[16] S. Liu, Int. J. Quantum Chem. 106, 1762 (2006).

[17] E. Clementi and C. Roetti, Roothaan-Hartree-Fock Atomic Wavefunctions (Academic, New York, 1974).

[18] J. Cioslowski, Phys. Rev. A 39, 378 (1989).

[19] J. Cioslowski, B. B. Stefanov, and A. Tan, J. Chem. Phys. 103, 6093 (1995).

[20] A. Holas and N. H. March, J. Mol. Struct.: THEOCHEM 315, 239 (1994).

[21] M. Hoffmann-Ostenhof, T. Hoffmann-Ostenhof, and W. Thirring, J. Phys. B 11, L571 (1978).

[22] A. Holas and N. H. March, Phys. Rev. A 51, 2040 (1995).

[23] I. A. Howard, N. H. March, J. A. Alonso, N. A. Cordero, and V. E. van Doren, Chem. Phys. Lett. 343, 166 (2001).

[24] N. H. March, Proc. Cambridge Philos. Soc. 48, 665 (1952).

[25] C. Carter, Proc. R. Soc. London, Ser. A 235, 321 (1956). 